\title{
Effectiveness of Novel Ankle Prophylactic Compared With Lace-Up Brace or Tape
}

\author{
Wyatt D. Ihmels, Kayla D. Seymore, and Tyler N. Brown
}

\begin{abstract}
Context: Conventional ankle prophylactics restrict harmful ankle inversion motions that lead to injury. But these existing prophylactics also limit other ankle motions, potentially leading to detriments in functional joint capacity. The ankle roll guard (ARG) may alleviate the prevailing issues of existing ankle prophylactics and prevent harmful ankle inversion, while allowing other joint motions. Objective: This technical report sought to compare the ARG's ability to prevent ankle inversion, but not restrict other ankle motions with existing prophylactics. Design: Repeated-measures study. Setting: Motion capture laboratory. Participants: Thirty participants. Intervention: Each participant had dominant limb ankle kinematics recorded during 5 successful trials of a sudden inversion event and 30-cm drop landing task with each of 4 conditions (ARG, ASO ankle stabilizer [brace], closed-basket weave athletic tape [tape], and unbraced [control]). Main Outcome Measures: Peak ankle inversion angle, range of inversion motion (ROM), and time to peak inversion during the sudden inversion event, and ankle plantar- and dorsiflexion ROM during the drop landing were submitted to a 1-way repeated-measures analysis of variance to test the main effect of prophylaxis. Results: Participants exhibited greater inversion ROM with control compared with tape $(P=.001)$, and greater plantar- and dorsiflexion ROM with ARG and control compared with brace $(P=.02, P=.001)$ and tape $(P=.02, P<.001)$. It took significantly longer to reach peak ankle inversion with brace and tape compared with ARG $(P<.001, P=.001)$ and control $(P=.01, P=.01)$. No significant difference in peak ankle inversion was observed between any condition $(P>.05)$. Conclusion: The ARG may prevent ankle inversion angles where injury is thought to occur (reportedly $>41^{\circ}$ ), but is less restrictive than existing prophylactics. The less restrictive ARG may make its use ideal during rehabilitation as it allows ankle plantar- and dorsiflexion motions, while preventing inversion related to injury.
\end{abstract}

Keywords: ankle sprain, ankle inversion, musculoskeletal injury

Ankle sprain, the most common sport and recreational injury, ${ }^{1}$ usually occurs from sudden joint inversion (ie, sole of the foot directed inward) greater than $30^{\circ} .{ }^{2}$ To prevent these injuries, health practitioners and athletes use prophylactic products, such as a laceup brace or athletic tape, to add mechanical joint stability. Typically, these prophylactic products seek to restrict ankle inversion, but not other joint motions (such as plantar- and dorsiflexion). Ankle prophylactic products are reportedly effective at reducing the frequency of injury by significantly altering static and dynamic ankle position, reducing maximum ankle inversion angle and velocity, and increasing the time to reach peak ankle inversion when compared with unbraced (control) ankles. ${ }^{3-5}$ However, the effectiveness of ankle prophylactics to prevent ankle injury and/or reinjury is reportedly dependent on specific device design. ${ }^{6}$

Two common ankle prophylactics include nonelastic athletic tape and lace-up brace. Nonelastic tape is typically applied in a closed-basket weave pattern around the ankle to provide mechanical stability. Lace-up braces generally provide stability with Velcro straps and/or laces, similar to a high top shoe, and/or rigid plastic supports attached to a nylon sheath covering the ankle. To reduce injury frequency, nonelastic tape and lace-up braces reportedly decrease peak ankle inversion and increase time to peak ankle inversion compared with an unbraced ankle up to $8^{\circ}$ and $15 \%$, respectively. ${ }^{5,6}$ Although both ankle prophylactics effectively reduce ankle inversion and subsequent injury, they reportedly restrict other ankle joint motions, including plantar- and dorsiflexion, during

The authors are with the Department of Kinesiology, Boise State University, Boise, ID, USA. Brown (tynbrown@boisestate.edu) is corresponding author. sports-related tasks, like the drop landing. Ankle prophylactics reportedly decrease plantar- and dorsiflexion motions up to $11^{\circ}$ during a drop landing. ${ }^{7}$ Restricting ankle motion may fail to address specific functional or mechanical postinjury impairments that predispose an individual to reinjury and joint instability. ${ }^{8}$

The ideal ankle prophylactic would not restrict other typical joint motions, rather only prevent the excessive ankle inversion thought to produce injury. ${ }^{1}$ With that in mind, an innovative prophylactic product — ankle roll guard (ARG) — was recently patented and designed to alleviate the prevailing issues of existing ankle prophylactic products. The ARG's novel design supposedly prevents excessive ankle inversion with a lightweight buttress on the lateral aspect of the shoe, but allows the user to maintain "other" ankle motions. However, ARG currently lacks verifiable and independent scientific data on the effectiveness of their prophylactic product. This technical report sought to quantify and compare the ARG's effectiveness to prevent excessive ankle inversion, but not constrain "other" typical joint motions with existing prophylactic products (both nonelastic tape and lace-up brace).

\section{Methods}

Thirty (15 males and 15 females) participants (21.7 [2.9] y, 1.7 [0.1] $\mathrm{m}$, and 72.5 [11.7] $\mathrm{kg}$ ) had ankle kinematics quantified during a sudden inversion event (Figure 1) and drop landing task (Figure 2). For the sudden inversion, participants stood on a wooden platform that contained side-by-side trap doors. Then, a researcher removed the mechanical support of 1 trapdoor, allowing the door to be rotated $30^{\circ}$ producing a sudden ankle inversion. For 
the drop landing task, participants stepped off a 30-cm plyometric box and landed on each foot simultaneously. Each participant performed both tasks with 4 conditions (ARG-Armor1, braceMedspec ASO Ankle Stabilizer [Medical Specialties Inc, Charlotte, $\mathrm{NC}$ ], tape - closed-basket weave athletic tape, and controlunbraced). Both the task and condition testing sequence were randomly assigned to each participant prior to beginning the study.

During testing, participants performed 5 successful trials for each task with every condition. During each trial, participants had dominant limb ankle biomechanics quantified from the 3D trajectories of 32 retroreflective skin markers. Marker trajectories were recorded with 8 high-speed (240 fps) optical cameras (Vicon, Oxford, United Kingdom). The recorded marker trajectories were filtered with low-pass fourth-order Butterworth filter at 12-Hz cutoff frequency and then processed to solve joint rotations in Visual 3D (v6.00; C-Motion, Rockville, MD) and expressed relative to participant's anatomical position. Ankle kinematics were time normalized from $0 \%$ to $100 \%$ of each event and resampled at $1 \%$ increments $(\mathrm{N}=101)$. During the sudden inversion, event start was defined as the first instance ankle inversion exceeded 2 SDs above baseline (anatomical position), and event end was defined as peak ankle inversion plus 20 frames. During drop landing, event start was defined as initial contact (when ground reaction force first exceeds $10 \mathrm{~N}$ ) and event end defined as weight acceptance (when ground reaction force stabilizes at body weight).
Ankle kinematics selected for statistical comparison included peak ankle inversion angle, range of inversion motion (ROM; peak angle-initial angle), time to peak ankle inversion for the sudden inversion, and range of plantar- and dorsiflexion motions for the drop landing. Each variable was submitted to a 1-way repeated-measures analysis of variance to test the effect of each condition (ARG, brace, control, and tape). A Bonferroni correction procedure was used for pairwise comparisons. All statistical analyses were performed using SPSS software (version 24.0; IBM, Armonk, NY) with alpha level at .05.

\section{Results}

There was a significant main effect of condition on inversion ROM $(P<.001)$ and time to peak ankle inversion $(P<.001)$, but not peak inversion angle $(P=.38)$ during the sudden inversion (Table 1 and Figure 1); and a significant main effect of condition for plantarand dorsiflexion ROM $(P<.001)$ during the drop landing (Table 1 and Figure 2). Participants exhibited greater inversion ROM with control compared with tape $(P=.001)$, as well as greater plantar- and dorsiflexion ROM with ARG and control compared with brace $(P=.02, P=.001)$ and tape $(P=.02, P<.001)$. It took significantly longer to reach peak ankle inversion with brace and tape compared with $\mathrm{ARG}(P<.001, P=.001)$ and control $(P=.01$, $P=.01)$.

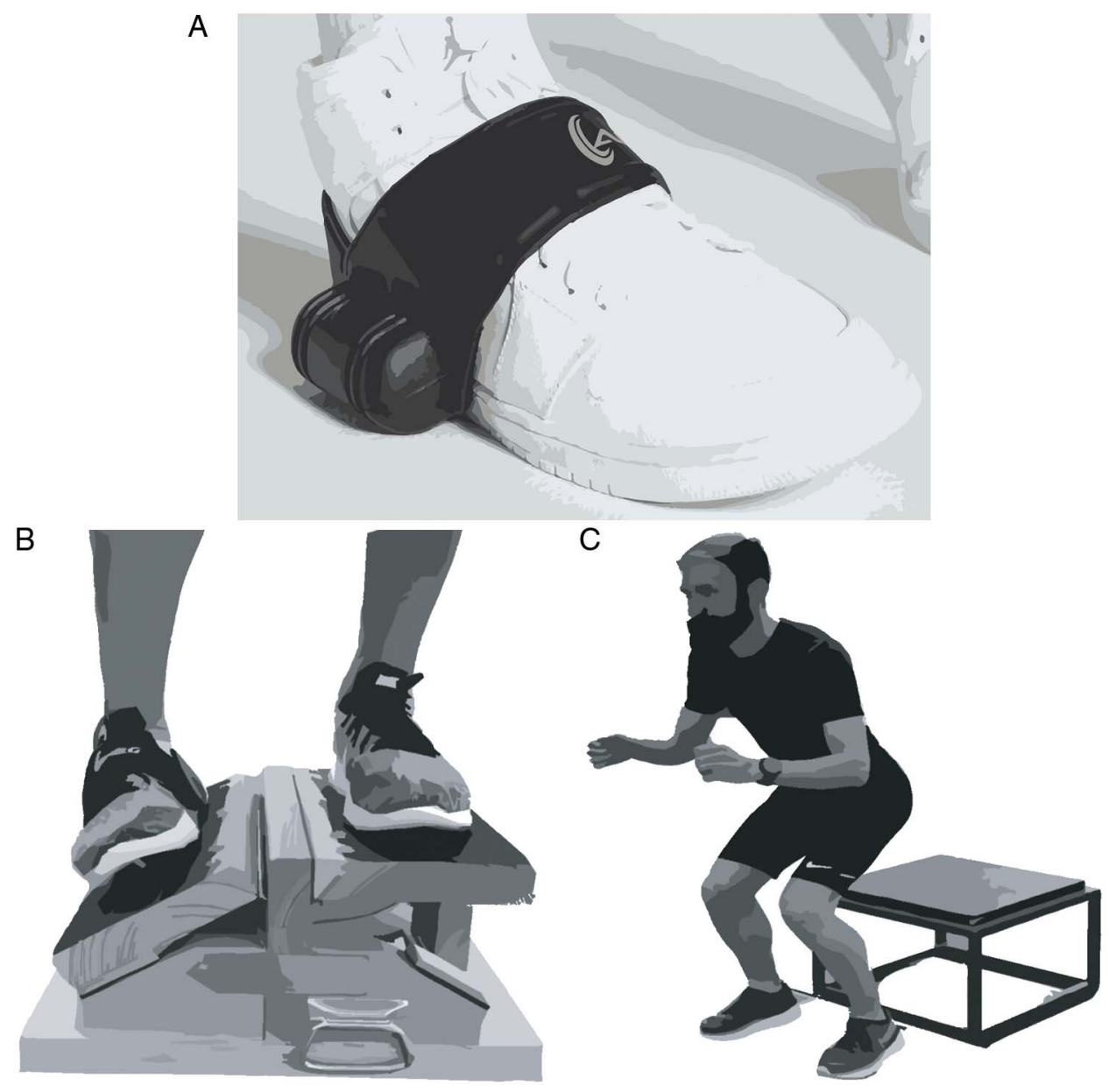

Figure 1 - Depicts the ankle roll guard (A), and the experimental setup for the sudden inversion event with the participant's ankle inverted (B) and drop landing $(\mathrm{C})$. 
A

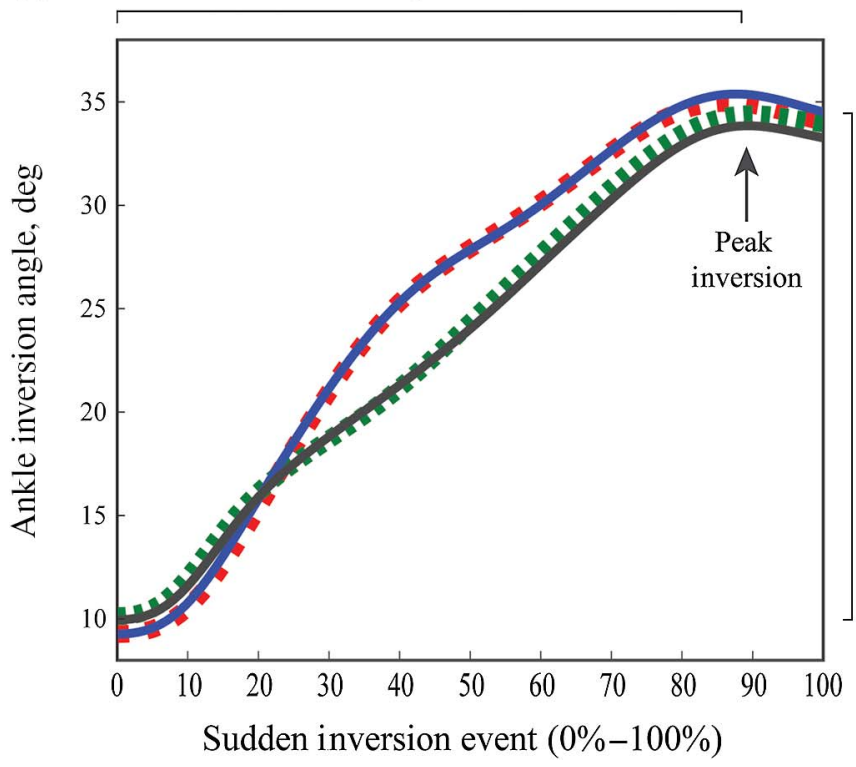

B

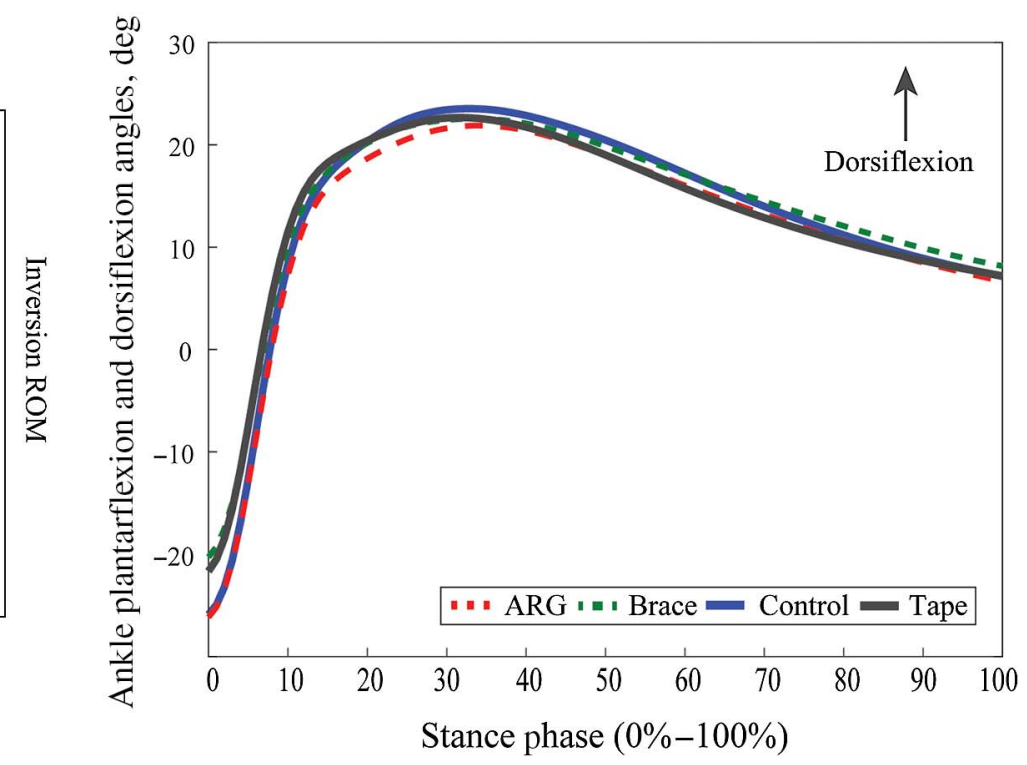

Figure 2 - Depicts mean (SD) ankle inversion (A) and plantar- and dorsiflexion angles (B) recorded for each condition (ARG, brace, control, and tape) during the sudden inversion event and landing phase (0\%-100\%) of the drop landing task, respectively. ARG indicates ankle roll guard; ROM, range of inversion motion.

\section{Table 1 Mean (SD) Ankle Kinematics Quantified During the Sudden Inversion and Drop Landing for Each Prophylactic (Ankle Roll Guard, Brace, Tape, and Control)}

\begin{tabular}{|c|c|c|c|c|}
\hline & \multicolumn{3}{|c|}{ Sudden inversion } & \multirow{2}{*}{$\begin{array}{c}\text { Drop landing } \\
\text { Range of plantar } \\
\text { flexion, deg }\end{array}$} \\
\hline & $\begin{array}{c}\text { Peak ankle } \\
\text { inversion, deg }\end{array}$ & $\begin{array}{l}\text { Range of ankle } \\
\text { inversion, deg }\end{array}$ & $\begin{array}{c}\text { Time to peak } \\
\text { inversion, } s\end{array}$ & \\
\hline Ankle roll guard & $35.1(4.6)$ & $26.1(3.1)$ & $0.19(0.04)$ & $49.5(9.5)$ \\
\hline Brace & $34.7(4.8)$ & $24.9(3.0)$ & $0.24(0.06)$ & $44.7(8.0)$ \\
\hline Control & $35.7(5.4)$ & $26.6(4.1)$ & $0.20(0.04)$ & $50.5(8.2)$ \\
\hline Tape & $34.2(5.3)$ & $24.4(4.2)$ & $0.23(0.04)$ & $45.4(6.9)$ \\
\hline
\end{tabular}

\section{Discussion}

The ARG may prevent excessive ankle inversion, but not as effectively as "more" restrictive brace and tape. During the sudden inversion, participants exhibited peak ankle inversion of $35.1^{\circ}$, $34.7^{\circ}$, and $34.2^{\circ}$ with the ARG, brace, and tape, respectively. Each ankle prophylactic prevented peak ankle inversion from exceeding $36^{\circ}$, or $5^{\circ}$ below where an injury occurred during a realistic sports maneuver. ${ }^{9}$ But, in contradiction with previous experimental evidence, ${ }^{3,4}$ no significant difference in peak inversion was observed between tested conditions, or between each prophylactic and the unbraced control. While the reason for the discrepancy is not immediately evident, the peak inversion angle currently exhibited during the control condition was $4^{\circ}$ smaller than previously reported. ${ }^{4}$ Considering ankle prophylactics, including the brace and tape presently tested, effectively reduce the frequency of ankle injury, the small reductions in peak ankle inversion $\left(\sim 0.6^{\circ}\right.$ to $\left.1.5^{\circ}\right)$ during the sudden inversion event may be clinically significant. All tested ankle prophylactics, ARG included, may provide mechanical stability to prevent joint inversion that leads to ligament damage and injury at the ankle, but do not meet a priori standards for statistical significance. Further study is warranted to determine whether ARG provides clinically significant reduction in peak ankle inversion compared with control or other ankle prophylactic design.

The ARG may limit harmful peak ankle inversion angles, yet may not provide the mechanical restriction of other prophylactics. During the sudden inversion, brace and tape provided $1.7^{\circ}$ and $2.2^{\circ}$ more restriction of ankle inversion ROM compared with control, whereas with the ARG, participants exhibited a nonsignificant increase of $1.2^{\circ}$ and $1.7^{\circ}$ in inversion ROM compared with the brace and tape. Furthermore, participants took significantly longer to reach the peak inversion with both brace $(0.24 \mathrm{~s})$ and tape (0.23 s) compared with ARG (0.19 s) during the sudden inversion. The increased restriction provided by the brace and tape may afford the user sufficient time to coordinate a neuromuscular response to effectively limit inversion motions and lengthen the time to reach peak ankle inversion, subsequently decreasing injury risk. ${ }^{10}$

Ideal ankle prophylactic design would not restrict typical joint motions, rather only prevent motions related to ligament damage and injury. ${ }^{1}$ Each tested prophylactic prevented excessive ankle inversion thought to lead to injury, but only the ARG allowed typical plantar- and dorsiflexion motions, while restricting ankle inversion. During the drop landing, participants exhibited $\sim 5^{\circ}$ greater plantar- and dorsiflexion ROM with the ARG compared 
with brace and tape; however, no difference was evident between ARG and control (approximately $50^{\circ}$ of total plantar- and dorsiflexion motions with each condition). This restriction of ankle motion may fail to address specific functional or mechanical postinjury impairments, that is, altered reflexes, neuromuscular control, or muscular weakness, increasing likelihood of joint instability development. ${ }^{8}$ Therefore, individuals who suffer an ankle sprain may benefit from ARG use during rehabilitation. During rehabilitation from initial ankle sprain, ARG use may allow the user typical ankle plantar- and dorsiflexion motions to decrease likelihood of functional impairment of the joint and instability development, while providing the restraint necessary to prevent excessive ankle inversion and injury during the healing process.

In conclusion, the ARG may provide the mechanical restriction to prevent ankle inversion angles that lead to injury (reportedly greater than $\left.41^{\circ}\right) .{ }^{9}$ Yet the ARG is less restrictive than brace and tape, and did not exhibit similar reductions in inversion ROM and time to peak inversion as those prophylactics. The ARG was the only prophylactic to allow typical ankle plantar- and dorsiflexion motions, while restricting excessive ankle inversion. The ARG's design may provide restraint to prevent injury during the healing process, but its limited mechanical restriction may make its use ideal during rehabilitation from initial injury, rather than as an injury prevention device.

\section{Acknowledgments}

This work was supported by Idaho Global Entrepreneurial Mission (IGEM) Award from the State of Idaho's Department of Commerce. The authors would like to thank the CAES Technical Assistance Program for the design and fabrication of the wooden platform.

\section{References}

1. Garrick JG, Requa RK. Role of external support in the prevention of ankle sprains. Am J Sports Med. 1973;5(5):200-203.
2. Norkus SA, Floyd RT. The anatomy and mechanisms of syndesmotic ankle sprains. J Athl Train. 2001;36(1):68-73. PubMed ID: 16404437

3. Anderson DL, Sanderson DJ, Hennig EM. The role of external nonrigid ankle bracing in limiting ankle inversion. Clin J Sport Med. 1995;5(1):18-24. PubMed ID: 7614076 doi:10.1097/ 00042752-199501000-00004

4. Eils E, Rosenbaum D. The main function of ankle braces is to control the joint position before landing. Foot Ankle Int. 2003;24(3):263268. PubMed ID: 12793492 doi:10.1177/107110070302400312

5. Hall EA, Simon JE, Docherty CL. Using ankle bracing and taping to decrease range of motion and velocity during inversion perturbation while walking. J Athl Train. 2016;51(4):283-290. PubMed ID: 27111586 doi:10.4085/1062-6050-51.5.06

6. Zhang S, Wortley M, Chen Q, Freedman J. Efficacy of an ankle brace with a subtalar locking system in inversion control in dynamic movements. J Orthop Sports Phys Ther. 2009;39(12):875-883. PubMed ID: 20026883 doi:10.2519/jospt.2009.3125

7. Cordova ML, Takahashi Y, Kress GM, Brucker JB, Finch AE. Influence of external ankle support on lower extremity joint mechanics during drop landings. J Sport Rehabil. 2010;19(2):136-148. PubMed ID: 20543215 doi:10.1123/jsr.19.2.136

8. Hertel J, Corbett RO. An updated model of chronic ankle instability. J Athl Train. 2019;54(6):572-588. PubMed ID: 31162943 doi:10. 4085/1062-6050-344-18

9. Fong DTP, Hong Y, Shima Y, Krosshaug T, Yung PSH, Chan KM. Biomechanics of supination ankle sprain: a case report of an accidental injury event in the laboratory. Am J Sports Med. 2009;37(4):822-827. PubMed ID: 19188559 doi:10.1177/ 0363546508328102

10. Ricard MD, Sherwood SM, Schulthies SS, Knight KL. Effects of tape and exercise on dynamic ankle inversion. J Athl Train. 2000;35(1): 31-37. PubMed ID: 16558605 